Service social

\title{
La recherche-action en santé communautaire, en travail social et en éducation : une nouvelle pratique ou un alibi pour professionnel?
}

\author{
Laurent Chambaud, Robert Mayer et Gabrielle Richard
}

Volume 35, numéro 1-2, 1986

Recherche - Action - Évaluation

URI : https://id.erudit.org/iderudit/706300ar

DOI : https://doi.org/10.7202/706300ar

Aller au sommaire du numéro

Éditeur(s)

École de service social de l'Université Laval

ISSN

1708-1734 (numérique)

Découvrir la revue

Citer cet article

Chambaud, L., Mayer, R. \& Richard, G. (1986). La recherche-action en santé communautaire, en travail social et en éducation : une nouvelle pratique ou un alibi pour professionnel? Service social, 35(1-2), 158-187.

https://doi.org/10.7202/706300ar 
Chambaud, Laurent, conseiller médical au Département de santé communautaire de l'Hôpital du Haut-Richelieu.

MAYER, Robert, professeur à l'École de service social de l'Université de Montréal.

RiCHARD, Gabrielle, agente de recherche au Département de santé communautaire de l'Hôpital du Haut-Richelieu.

\section{La recherche-action en santé communautaire, en travail social et en éducation : une nouvelle pratique ou un alibi pour professionnels ? 1}

\section{Laurent Chambaud Robert Mayer Gabrielle Richard}

La recherche-action est devenue, au cours des dernières années, un thème à la mode au Québec. Elle s'est développée dans divers domaines, notamment dans les secteurs de la santé et du travail social. Un diagnostic d'ensemble a récemment été porté sur l'évolution de la recherche-action au Québec. Ainsi, Goyette et Lessard-Hébert (1985) formulent deux constats à propos de la recherche-action :

"Le premier concerne l'importance quantitative grandissante des pratiques de recherche-action et les questionnements qu'elle suscite quant à sa notion, ses fondements, sa valeur et ses outils méthodologiques. Le deuxième constat est qu'il n'y a pas de réponse unique et simple à ces questions puisqu'il existe une diversité de définitions, de conceptions et de pratiques de recherche-action.» (1985: 22).

On doit par ailleurs constater que la recherche-action exerce, depuis quelques années, un certain attrait dans le secteur sociomédical. Comme l'ont signalé Le Gal et Martin : 
"La séduction que cette démarche opère tant chez les travailleurs sociaux que chez les décideurs et responsables d'institutions de l'action sociale, renvoie plus particulièrement à sa dimension collective et consultative. Elle présente également pour ces mêmes auteurs, l'avantage de développer une conception professionnelle de la recherche sociale, permettant de dépasser l'habituel placage d'une recherche traditionnelle et universitaire, souvent trop distanciée et trop théorique..." (1983: 67).

De même, Dubost (1984: 9) a noté que l'imprécision de la notion de recherche-action a pour effet de renforcer son caractère un peu mystérieux, "son côté miroitant, anti-conformiste, propre à éveiller l'espoir de réduire les oppositions entre les deux termes... " et ce qui a pour effet, précise-t-il, de faire

" fantasmer facilement une partie des formateurs, des psychosociologues, des travailleurs sociaux, des militants mais aussi certains décideurs des appareils technocratiques qui souffrent du fossé qui s'est creusé entre chercheurs et praticiens et qui sentent leurs exigences de rationalité mises à mal par cette évolution. " (1983: 17).

Nous assistons également à une production relativement abondante de textes théoriques sur la recherche-action et qui portent sur des problèmes de définition, de spécificité méthodologique ou d'explication épistémologique. Tous ces éléments sont certes très importants mais ils laissent le lecteur sur sa faim, qu'il soit intervenant, chercheur ou autre, car tous les problèmes concrets liés à l'exercice de la recherche-action sont posés avec une acuité croissante dans la pratique mais sont à peine abordés dans la littérature. Parmi ces problèmes, celui de la relation entre les principaux acteurs (population, intervenant et chercheur) nous paraît central. Le thème principal de ce texte est précisément d'examiner d'une façon générale la nature des rapports entre les principaux acteurs dans le processus de recherche-action, et plus particulièrement la place des "usagers" ou de la population dans ce processus. Pour ce faire, nous avons, dans un premier temps, centré notre analyse sur un domaine particulier, celui de la santé, en nous basant sur une analyse de contenu de certains comptes rendus de recherches-actions en santé québécoise.

Nous n'avons pas la prétention de pouvoir séparer le bon grain de l'ivraie, les vraies recherches-actions des fausses car, comme l'a signalé Martin (1985: 130), il n'y a heureusement pas "de marque déposée » du genre; nous avons donc opté pour un principe assez simple et démocratique, à savoir : " est recherche-action ce qui se dit rechercheaction » (Martin, 1985 : 127). Plus brièvement ensuite nous avons voulu voir si les tendances que l'on retrouve dans le secteur de la santé se 
retrouvaient également dans d'autres secteurs, tels que ceux du travail social et de l'éducation. Soulignons toutefois qu'il ne s'agit pas ici d'une analyse systématique et exhaustive de la production récente sur la recherche-action, comme il commence à s'en faire chez nous (Goyette et Lessard-Hébert, 1985: Gélinas et Brière, 1985) ou ailleurs (Martin, 1986). Nos ambitions sont plus modestes; il s'agit plutôt d'un « coup de sonde" afin de vérifier certaines intuitions ou impressions. Notre hypothèse de départ est celle-ci : il semble se dessiner un décalage entre les discours théoriques sur la recherche-action et les pratiques réelles et concrètes en ce qui concerne la participation de la population.

\section{La recherche-action en santé}

\section{La santé : un concept mouvant et tentaculaire}

S'interroger sur la recherche-action en santé c'est, bien entendu, poser tout le problème de la définition et de la multiplicité des discours entourant la notion même de recherche-action et des possibilités de cette approche dans les différents champs d'études et disciplines professionnelles. C'est aussi être confronté à l'évolution récente du concept de santé et à l'élargissement de ce champ d'investigation. En fait, une des premières difficultés rencontrées fut de délimiter ce que nous entendions par le terme recherche-action en santé ; il nous fallait tracer une ligne entre les recherches-actions appartenant à d'autres "spécialités». Ce qui semblait l'évidence même s'est compliqué par les transformations profondes qu'a connu le concept de santé, dépassant désormais largement la simple définition "d'absence de maladie». Les nouvelles notions de santé globale et d'approche holistique traduisent ce changement de conception où le thème de la relation de l'homme à son environnement prend toute son importance. Nous assistons ainsi à une pénétration - certains diront une restitution - du champ de la santé dans l'ensemble de la vie sociale.

Cette évolution rend possible deux lectures différentes de l'interpénétration du social et de la santé : d'une part, la santé se "socialise", puisque des déterminants autres que médicaux sont pris en considération dans l'analyse des problèmes de santé (situation socioéconomique, aspects environnementaux). Cette vision amène donc à identifier des types d'interventions non médicales (action politique, action communautaire, décisions économiques) pour résoudre des problèmes de santé. 
L'évolution à laquelle nous faisons allusion est aussi manifeste à la lecture des principes de base adoptés en 1978 lors de la conférence d'Alma Ata sur les soins de santé primaires, et ratifiés par cent trentequatre pays, dont le Canada : la Conférence réaffirme avec force que la santé, qui est un état de complet bien-être physique, mental et social et ne consiste pas seulement en l'absence de maladie ou d'infirmité, est un droit fondamental de l'être humain, et que l'accession au niveau de santé le plus élevé possible est un objectif social extrêmement important qui intéresse le monde entier et suppose la participation de nombreux secteurs socio-économiques autres que celui de la santé.

D’autre part, des événements de nature sociale sont récupérés dans le champ de la santé permettant de justifier des interventions sanitaires. C'est d'ailleurs ce que prétend Zola: "La métaphore de la santé et de la maladie sert de plus en plus de variable explicative, sinon d'explication en elle-même de tout un train de problèmes sociaux " (1981: 46). Barbara Ehrenreich constate ce même phénomène lors du développement du scientisme aux États-Unis au début du siècle : "Les problèmes d'ordre social et dont la solution devrait donc être politique furent transformés en problèmes d'ordre scientifique, dont la solution devenait technologique" (1981: 95). Le questionnement actuel autour des nouvelles technologies de reproduction vient nous rappeler que ce débat est loin d'être clos.

Nous nous retrouvions donc face à un univers de santé mouvant et tentaculaire dont il était difficile de préciser les limites et les contours. Pour faire face à cet obstacle, nous avons retenu une définition très concrète en tenant compte, d'abord et avant tout, de la présence des professionnel(le)s de la santé. Nous postulions donc que le champ d'intervention de ces professionnels(les) définissait l'étendue des pratiques de santé. Selon cette proposition, si un problème était investi par le corps de ces spécialistes, la problématique devenait sanitaire et l'intervention justifiée (ou justifiable); en dehors de cela, il s'agissait d'une pratique sociale ${ }^{2}$.

\section{La recherche-action en santé : analyse des discours et des pratiques}

Les rapports entre recherche et intervention sont, dans le domaine de la santé publique, sujets à questionnements depuis une dizaine d'années. S'appuyant sur plusieurs auteurs, en majorité américains, Daab et Abenhaïm (1985) constatent un "hiatus" entre l'intérêt des chercheurs et les questions d'actualité. Ils avancent plusieurs explications possibles reliées à l'idéologie, à l'institution, aux difficultés 
de communication entre producteurs et utilisateurs de connaissances ou aux contraintes liées à la méthode scientifique. Navarro, dans un article traitant des liens entre science et idéologie, se base sur des expériences européennes pour définir un type de recherche qui « place le chercheur dans une relation sociale différente avec le sujet de l'étude" (1980: 546). Pour cet auteur, de par leur position idéologique, les professionnels favorisent une forme de production de connaissance conforme au modèle bourgeois et « ils doivent changer non seulement leur rôle [de leaders à assistants] mais aussi leurs méthodes de travail et le contexte social et politique dans lequel ils exercent $"$ (p. 596).

Au Québec, il semble que la recherche-action soit le lieu privilégié et le résultat d'interrogations sur les liens possibles et nécessaires entre la recherche et l'intervention dans le domaine de la santé communautaire. En fait, ce questionnement se retrouve d'une manière très concrète dans trois des quatre comptes rendus de recherches recensés à travers le Répertoire des recherches en santé au Québec (GRIS, 1985). Ces textes font effectivement état d'une réflexion sur le fossé existant entre la théorie et la pratique dans le domaine de la santé communautaire, sur l'insatisfaction à l'égard des études de besoins et des démarches de programmation traditionnelles qui interviennent sur et non avec la population.

Les expériences demeurent cependant peu nombreuses, isolées, et surtout ne permettent pas d'élaborer une réflexion sur les caractéristiques spécifiques à la recherche-action en santé. C'est pourquoi le document produit récemment par l'Institut national de la santé et de la recherche médicale (INSERM) en France nous semble particulièrement important à relever car il nous permet d'élaborer et, dans une certaine mesure, de vérifier l'hypothèse suivante : dans la recherche-action en santé, nous assistons à une conception particulière de la définition et du rôle des partenaires. Pour ses auteurs, elle

"est un processus dynamique et court conduisant à l'élaboration, à la mise en ouvre et à l'évaluation d'une "action" (ou d'un ensemble d"“actions"), dans le but de préservation et/ou d'amélioration de la santé. Elle unit dans la même tâche deux types de professionnels: un ou plusieurs chercheurs et un ou plusieurs "acteurs" (praticiens de terrain, décideurs) n'appartenant pas à la communauté scientifique. Ce processus concerne la communauté et implique un consensus entre les différents partenaires sur les objectifs et les moyens. » (P. 19.)

La recherche-action en santé poursuit trois grands objectifs: contribuer à l'amélioration de la santé de la population; répondre aux problèmes concrets que se posent les praticiens de terrain et ainsi modifier leur pratique; de plus, pour l'INSERM, elle est également un 
outil de valorisation sociale permettant de prendre en compte, tant les résultats de recherches que les demandes de recherches à l'extérieur de I'Institut (pp. 21-22). Un dernier point nous paraît crucial : I'analyse des partenaires.

"Ceux-ci appartiennent à diverses catégories de professionnels que pour simplifier on peut classer en "chercheurs" [ceux dont le métier est de faire institutionnellement de la recherche] et en "acteurs." " (p. 25). "Si les acteurs peuvent être des usagers ou leurs représentants, il est ici clair que ceux-ci sont le plus souvent écartés du processus de recherche-action. Témoin, ce commentaire: "Parmi les partenaires importants de la recherche-action en santé figurent les 'usagers'. Ils représentent la cible des autres partenaires, leur position est très inconfortable. Est-ce la raison pour laquelle il en a finalement été très peu question?" "(p. 30).

La définition et le rôle des acteurs, tels que définis par les auteurs du présent document, prend donc une coloration bien précise: l'acteur de la recherche n'est pas la communauté aux prises avec un problème de santé mais le professionnel de la santé dont il faut "modifier la pratique». La transformation de l'état de santé d'une population passe donc par une transformation de la pratique des professionnels. Quant aux usagers, ils deviennent (ou plutôt restent) la cible de cette nouvelle entité chercheurs/professionnels, bien que leur soit octroyé le titre, très honorifique, de partenaires. Cet élément est d'ailleurs appuyé par les résumés de communications qui sont reproduits en deuxième partie du document de I'INSERM: des six recherches-actions en santé présentées dans ce document, aucune n'accorde à la communauté cible un rôle actif quelconque, le "processus de recherche-action " étant centré sur les professionnels de la santé.

Si nous nous penchons maintenant sur cette situation au Québec, un constat analogue peut être fait dans le domaine de la santé communautaire. En effet, trois des quatre recherches-actions en santé que nous avons retenues mettent l'accent sur une sensibilisation des professionnels ou sur une formation et modification de la pratique des intervenant(e)s. Ainsi, dans "l'expérimentation d'une intervention visant les pratiques alimentaires de pré-adolescents au déjeuner» (Département de santé communautaire du CHUL, 1984) un des trois objectifs était de : "fournir aux professionnels et éducateurs impliqués dans la promotion de la santé un cadre de compréhension élargi des phénomènes qui entourent les pratiques alimentaires" (p. 3).

Dans Le collectif garderies: une expérience d'éducation des adultes en santé communautaire, (Caron, 1982) l'auteur scrute le rôle de 
l'équipe de santé communautaire auprès des milieux de garde à l'enfance en réalisant un projet exploratoire. L'objectif de cette recherche était d': "Évaluer le groupe de travail comme méthode d'apprentissage pour permettre aux professionnels de la santé de se donner la formation requise à cette nouvelle tâche" (l'action communautaire) (p. 42).

Enfin, dans la recherche-action dans le secteur Saint-René-Goupil du quartier Saint-Michel à Montréal (intervention communautaire en santé de l'Hôpital du Sacré-Cour, 1982), la commande initiale était de "faire une étude des besoins de 0-1 an, afin de mieux adapter les activités du programme de santé maternelle et infantile aux besoins réels» (p. 2). Même si, au départ, on notait la volonté d'impliquer les ressources de la communauté qui interviennent auprès de la population et cette même population (p. 3), le constat final rejoint singulièrement les expériences françaises: "Nous pouvons dire maintenant que la recherche-action a permis surtout d'impliquer chercheurs et intervenants du département de santé communautaire. " (P. 56.) On peut également lire plus loin: "En troisième lieu, notre recherche-action n'a pas été jusqu'à impliquer véritablement la population du quartier." (P. 57.)

Quant à la quatrième recherche-action retenue, Le rapport des visites auprès des familles du sud-est asiatique, (Département de santé communautaire de l'Hôpital Sainte-Justine, 1981) l'objectif premier était "d'informer la clientèle sur les sources possibles de solutions à leurs problèmes" en passant par l'identification (au moyen de questionnaires) « des problèmes socio-sanitaires de la clientèle cible et "la connaissance de leurs caractéristiques démographiques et administratives» (p. 8). Nous sommes donc bien plus proches d'une analyse de besoins traditionnelle que d'un véritable processus de recherche-action.

Autre illustration de l'utilisation de la recherche-action comme processus dirigé essentiellement vers les intervenants : l'expérience de l'équipe d'intervention en réseau du Centre hospitalier Douglas (1982). Dans un document intitulé Réseau primaire et santé mentale: une expérience de recherche-action (1982), les auteurs définissent ainsi l'intérêt de la démarche: "Dans l'ensemble, on peut dire que la recherche-action s'avère une démarche utile et intéressante pour l'élaboration d'un modèle d'intervention, autant pour son produit [connaissances théoriques et pratiques] que par le processus de collaboration qu'elle suscite entre chercheurs et praticiens." (1982: 155.)

Parfois, malgré une volonté affirmée d'engager la communauté dans le processus de recherche-action, certaines expériences 
aboutissent à un constat d'échec. C'est le cas du "projet santé agricole " dans lequel: "... nous avons réussi ce tour de force de tenir la population agricole [les acteurs] à l'écart du projet et ce à deux niveaux : à l'écart du processus de recherche-action et à l'écart de la recherche. "(Chambaud et Richard, 1985 : 32.)

Une dernière expérience québécoise nous indique que le modèle dominant retrouvé dans le secteur institutionnel de la santé est également présent dans les milieux alternatifs. En effet, la rechercheaction avec le groupe "Alternative naissance" (Conte, Loncol et Regimbald, 1985) s'apparente à un diagnostic organisationnel dirigé vers les membres actifs de ce groupe afin "de les aider à atteindre l'autofinancement total» (p. 9). Il s'agit donc essentiellement d'une expérience de collaboration avec le noyau militant de ce groupe, mais qui n'implique pas les femmes ou les couples recevant des services ou faisant appel à Alternative naissance.

Nous sommes donc portés à croire que, dans le milieu institutionnel de la recherche en santé communautaire, au Québec, la rechercheaction est interprétée comme un processus d'acquisition de connaissances d'une communauté par les praticiens de terrain dans le but de modifier des pratiques ou d'adapter des programmes. Nous avons cependant relevé, dans la littérature, une exception à cette tendance. Le travail réalisé à l'Hôpital général de Québec, et rapporté dans un article, semble en effet emprunter une autre voie (Darveau-Fournier, 1985). Son but premier était « d'améliorer la qualité de vie des bénéficiaires et la satisfaction au travail des intervenants par un processus de formation continue». Un peu plus loin, on ajoutait que les principaux objectifs poursuivis visaient «à stimuler le développement d'attitudes qui favorisent une approche globale du bénéficiaire" et "à valoriser la contribution particulière des intervenants et des bénéficiaires au sein de l'équipe multidisciplinaire» (1985: 32). En effet, la méthodologie retenue, dit-on, fait appel à la participation de toutes les catégories de personnes formant la communauté de l'hôpital et, plus encore, on affirme que "sont considérés comme des experts toutes les personnes impliquées dans la vie de l'institution, y compris les bénéficiaires et leur famille ". À notre connaissance, cette recherche-action est unique dans le domaine de la santé communautaire.

\section{La santé au travail : une exception exemplaire dans le contexte québécois}

Il existe cependant un secteur, au Québec, où les expériences de recherche-action fournissent à la communauté subissant un problème 
de santé la possibilité d'uacter» la recherche: il s'agit de la santé au travail. Les deux expériences rapportées dans la Revue internationale d'action communautaire sont, à cet égard, extrêmement significatives, car toutes deux font intervenir un autre partenaire - le syndicat - qui prend une part active dans le processus en sensibilisant et mobilisant les travailleurs et travailleuses autour de la recherche.

Desnoyers et Mergler (1979), constatant "que l'amélioration des conditions de travail résulte généralement de luttes menées par les travailleurs et par leurs organisations syndicales " (p. 11), estiment que le rôle des scientifiques progressistes est de fournir une aide scientifique et technique à ces luttes. La recherche qu'ils ont effectuée auprès des travailleurs et travailleuses d'un abattoir de volailles en est une illustration. Hétu (1979) est particulièrement intéressant à cet égard lorsqu'il rapporte qu'une première expérience de recherche s'était déroulée avec deux intermédiaires: un organisme gouvernemental, bailleur de fonds sur le plan administratif, et une équipe de médecins progressistes, au niveau de la coordination avec les ouvriers. Or, affirme-t-il : « La distance que ces deux intermédiaires imposaient entre le syndicat et moi suffisait à me rassurer quant à la nature de mon intervention en tant que scientifique : beaucoup de recherche et peu d'action... " (1979: 22).

Le domaine de la santé au travail nous apparaît présenter deux particularités majeures qui "changent les règles du jeu» et imposent une vision différente de la recherche-action. Tout d'abord, le caractère récent de l'intérêt porté par les professionnels de la santé à ce domaine. Malgré une longue tradition de lutte des travailleurs et travailleuses pour améliorer leurs conditions de travail (Mergler et Desnoyers, 1981), les représentants du monde médical ne se bousculaient pas pour occuper ce champ; jusqu'en 1979, hormis quelques services de santé mis en place, payés et contrôlés par certaines grandes compagnies, le terrain était relativement vierge de tout professionnel de la santé. Cependant, depuis l'adoption de la Loi 17 sur la santé et sécurité au travail (1979) et dont le contenu a déjà été résumé ailleurs (Vézina, 1985), il semble se développer un intérêt croissant pour cette problématique, aussi bien du côté de la recherche - avec la création de l'Institut de recherche en santé et sécurité au travail - que de la pratique. Témoin, le développement de nouvelles disciplines: médecine en santé au travail, hygiène industrielle, ergonomie, etc.

Nouveau champ à investir, nouveau gâteau à partager... Ces nouveaux professionnels pourraient donc, selon notre hypothèse, contribuer à modifier la pratique de la recherche-action dans ce domaine. Mais, également, la santé au travail se doit de compter avec 
des « usagers » qui ne sont plus, comme dans les services traditionnels de santé, seuls, inorganisés et sans force revendicatrice; en effet il existe, du moins dans certaines entreprises, un partenaire qui représente - ou est censé représenter - les intérêts collectifs de ces travailleurs: le syndicat. Nous pensons que cet élément incite, et parfois même oblige, tant les intervenants que les chercheurs à considérer d'une autre manière leur "population cible".

Ainsi, l'absence relative de professionnels de la santé véritablement organisés et aussi la relative organisation des "bénéficiaires" des programmes de santé au sein de leurs associations syndicales pourraient conjointement inciter à penser autrement la rechercheaction au Québec.

\section{La place des acteurs : deux hypothèses explicatives}

Nous avons vu comment la recherche-action en santé situait les différents partenaires ; il serait donc plus exact de parler de " rechercheintervention" auprès de la population. L'exemple de la santé au travail nous indique deux hypothèses explicatives à ce phénomène :

- La première tient à la présence d'une quantité importante et diversifiée de professionnel(le)s de la santé. II existait, au Québec, en 1981: 13244 médecins, 2468 dentistes et plus de 95000 personnes travaillant dans des occupations subordonnées directement liées à la dispensation de services sanitaires (Dussault, 1985). Dit plus simplement, au Québec, une personne sur quarante vit de la santé 3 . Or, si nous reprenons la définition du processus de médicalisation défini par Freidson: "La profession médicale jouit d'un droit prééminent de déterminer ce qui constitue une maladie et tout ce qui en relève, indépendamment de sa capacité d'y remédier efficacement" (Freidson, cité dans : Zola, 1981, p. 39), il ne paraît pas incongru d'élargir cette définition au champ de la santé en général, qui apparaît comme un domaine balisé d'une manière très concrète par la présence des professionnel(le)s de la santé ${ }^{4}$. La recherche-intervention dans ce cadre viendrait donc légitimer ou modifier les pratiques professionnelles auprès de la population. Toute implication de la communauté ne se ferait que par l'intermédiaire des professionnel(le)s, relais obligatoires et valorisés (ce sont eux qui connaissent "le mieux" "leur" population).

- La deuxième explication porte sur le niveau d'organisation de la population. À la lumière des exemples que nous avons donnés, 
un fait semble se dégager : plus une communauté est organisée, moins elle a de risques de se faire organiser. Cette assertion, qui n'a rien d'original, n'en reste pas moins appropriée. En effet, il n'existe à peu près pas, dans le domaine de la santé, de mouvement de défense des usagers, mis à part quelques associations marginales. Les " patients » sont donc livrés, en ordre dispersé, aussi bien aux interventions des différents professionnels qu'aux expérimentations des chercheurs. Et, malheureusement, l'introduction du concept de recherche-action ne semble pas avoir modifié cette vision. Par contre, en santé au travail, la population cible, tout au moins dans les lieux où elle est organisée, dispose d'une voix. Dans ce cas, la situation s'inverse et la population passive peut devenir actrice de son changement, voire même commanditaire.

Pour nous assurer de la plausibilité de ces deux hypothèses explicatives, nous avons parcouru les pratiques de recherche-action dans d'autres domaines que la santé.

\section{La recherche-action en travail social}

\section{Le discours}

À titre comparatif, nous avons examiné le secteur du travail social en général et, notamment, le champ plus spécifique de la gérontologie sociale. Comme l'a signalé Martin (1985: 130), ces secteurs sont tout à fait exemplaires de la quête "d'alternative méthodologique ", et l'un et l'autre commencent à faire l'objet d'un inventaire critique, au Québec, alors que cette mise en ordre est passablement avancée en France. Ces domaines d'investigation sont caractérisés, à la fois par la présence de praticiens, par l'existence de problèmes sociaux à résoudre de façon urgente, et par le fait qu'ils proposent un équilibre entre une production autonome par des acteurs sociaux sur leurs propres pratiques et une production hétéronome effectuée sur les acteurs sociaux et leurs pratiques par des chercheurs extérieurs.

Nous assistons à un net intérêt des milieux du travail social pour la recherche sociale en général et la recherche-action en particulier. Les raisons d'un tel rapprochement sont multiples. Pour Bousquet (1984), cet intérêt s'explique notamment par les phénomènes suivants : la crise de l'État-providence et de ses politiques sociales; les débats sur la formation et les pratiques des travailleurs sociaux; et, finalement, l'évolution des sciences humaines. Ces diverses évolutions suscitent un 
intérêt pour la recherche et divers enjeux en découlent : personnel (l'espoir d'un meilleur statut) ; professionnel (le prestige scientifique de la profession); et de pouvoir (la capacité critique vis-à-vis le pouvoir). Mais, selon l'auteur, nous assistons à une division des professionnels : les uns s'opposent à la colonisation du travail social par des disciplines scientifiques et les autres ont peur de la non-scientificité des recherches faites par des praticiens/chercheurs.

Pour sa part, Autès explique que, si le travail social s'intéresse maintenant davantage à la recherche, c'est parce que celle-ci lui apparaît comme pouvant être une issue à des difficultés, voire des impasses:

"On pourrait certes analyser cette nouvelle conjoncture comme une étape dans le développement du travail social. Sans doute que l'accroissement du nombre de professionnels, le développement des formations supérieures, la multiplication des formes d'intervention et leurs contradictions, la remise en cause du coût du social dans une période de crise sont, parmi d'autres, des facteurs qu'il faudrait prendre en compte pour éclairer cette évolution. Comme si le travail social recherchait du côté de la recherche une nouvelle légitimité. » (1984: 16.)

Un peu dans la même veine, Martin explique l'intérêt grandissant des milieux de pratique vis-à-vis la recherche-action comme un moyen de pallier partiellement aux diverses critiques dont ils sont l'objet :

"le milieu professionnel, voyant s'effriter les repères traditionnels [discours de la bienfaisance, légitimité articulée à la promotion du bien-être par l'État-Providence] a répondu par une quête importante de repères. Ce qui semble de plus en plus manifeste aujourd'hui est le recours à la recherche, à la science, comme légitimité transcendante des pratiques. Plus qu'un recours à la recherche, le milieu professionnel manifeste un souci de maîtrise de ces productions, refusant le rapport de domination existant antérieurement vis-à-vis des chercheurs. Ce souci d'appropriation de la production de résultats scientifiques est d'ailleurs aujourd'hui renforcé par la nécessité devant laquelle se trouve le travail social de faire la preuve de l'efficacité de ses pratiques. " (1985: 134.)

À cet intérêt grandissant pour la recherche-action en travail social correspond un éventail d'applications très large. Il y a lieu de souligner également la très grande hétérogénéité des pratiques et des récits de recherche-action. S'il y a une diversité des pratiques, c'est qu'il y a aussi une diversité des conceptions méthodologiques. II n'y a pas en la matière de projet unique ni de dispositif méthodologique reconnu et admis de tous, mais bien différentes conceptions du travail de recherche-action et aussi différentes pratiques. Le champ de la 
recherche-action englobe des courants aussi hétéroclites que la méthode de conscientisation de Paulo Freire, l'analyse institutionnelle, l'intervention sociologique, etc. Dans un essai de clarification conceptuelle, Dubost (1983: 20) aboutit à la conclusion que la recherche-action n'est pas une, mais multiple et peut exprimer aussi bien une stratégie de recherche, d'action, d'existence, que d'analyse sociale. Au Québec, Rhéaume (1982) a précisé que la pratique de recherche-action s'est développée principalement dans les années soixante, en particulier dans les secteurs de l'animation sociale et de l'éducation.

À ce propos, certains n'ont pas manqué d'évoquer, avec une certaine pointe d'ironie (Wieviorka, 1980), l'enthousiasme mais aussi l'idéalisme des premiers chercheurs qui se sont précipités en milieux populaires supposément pour leur venir en aide suite à l'annonce, par l'État, de projets d'aménagement du territoire ou de "rénovation urbaine ». Le Québec a aussi connu ce phénomène. Ce fut d'abord la vogue des enquêtes-participation, dont une des plus célèbres fut celle du Bureau d'aménagement de l'Est du Québec (B.A.E.Q.). Dès 1963, le B.A.E.Q. entreprend des enquêtes impliquant la participation des citoyens afin d'élaborer un plan d'aménagement de la région. Ce projet va aussi incorporer à sa méthode de recherche et de planification des méthodes d'animation pour susciter la participation des populations locales.

Par la suite, cette double stratégie de recherche et d'animation sera largement appliquée dans plusieurs quartiers urbains des principales villes québécoises. Aux résidents des quartiers réunis en comités de citoyens s'ajoutent des universitaires, des stagiaires (service social), des leaders locaux (i.e. curés) qui apportent leur "expertise». Cette recherche "d'experts du peuple" traduit la volonté, chez plusieurs comités de citoyens, de se situer sur un pied d'égalité avec les technocrates gouvernementaux afin de leur faire valoir objectivement leur point de vue et en arriver possiblement à la décision la plus rationnelle possible. Rationalité et participation, tels sont en effet les maîtres-mots de l'époque.

L'évolution de la dernière décennie a modifié les règles du jeu. Nous assistons à une bureaucratisation croissante des institutions gouvernementales, du système scolaire ou des organismes des « affaires sociales ", de même qu'à une spécialisation et une professionnalisation des divers praticiens: organisateurs communautaires, travailleurs sociaux, etc. Le climat politique est venu perturber l'esprit de concertation et de participation qui caractérisait, au moins au plan idéologique, la période précédente. Par contre, la conjoncture de crise 
qui sévit depuis le début des années quatre-vingt a accentué l'acuité des divers problèmes sociaux et rend la recherche-action plus " populaire", ou tout au moins plus acceptable auprès des pouvoirs publics, dans la mesure où elle se veut plus pratique, plus concrète, plus préoccupée par la recherche de solutions. D'où la nécessité de bien distinguer les discours et les pratiques réelles.

Les travaux de Le Boterf $(1981,1983)$ ont très bien posé le problème de l'établissement de nouveaux rapports entre les divers participants d'une recherche-action. Ce dernier souligne notamment que, dans le modèle classique de la recherche scientifique, les résultats vont essentiellement aux commanditaires, parfois à la communauté scientifique "qui en fera un nouvel objet de recherche traité dans les colloques, séminaires, publications, etc. Cette production alimentera en retour, le travail des chercheurs" (1983: 40). Dans cette perspective, les "enquêtés" sont considérés comme des "cobayes" n'ayant pas à connaître l'aboutissement du travail dont ils sont l'objet. Cette conception de la recherche appartient à ce que certains ont appelé "les sciences de l'autre " et qui fonctionne "en supposant que l'essentiel du dire de l'autre n'est pas accessible à celui qui l'exprime" (1983: 41). Ceux qui s'efforcent de transformer le discours de l'autre en discours savant disposent donc d'un pouvoir institutionnel et méthodologique considérable.

Pour contrer ces limites et ces pièges, la majorité des auteurs estiment que la collaboration entre les partenaires de la rechercheaction repose sur la complémentarité, tout en préservant la spécificité de chacune des parties. Pour Le Boterf (1983: 45), le problème principal n'est pas la question de la suppression ou du retrait du chercheur, mais bien plutôt la question de la gestion de nouveaux rapports entre les experts et les acteurs. Mais toutes ces bonnes intentions que l'on retrouve dans la littérature "n'alimentent-elles pas qu'un discours imaginaire ou débouchent-elles vraiment sur des pratiques novatrices dans la production du savoir? ". S'agit-il vraiment d'une déprofessionnalisation de la recherche ou de la naissance de ce que Corpet (1982) a appelé une nouvelle «intelligentsia alternative"? Nous avons constaté précédemment que la population est peu présente dans les expériences de recherche-action en santé. Or, Martin (1985) a formulé un constat qui va dans le même sens à propos de la recherche-action en travail social. S'il existe une constante dans la littérature, précise-t-il, "elle réside dans le rapprochement d'acteurs différenciés: des praticiens, des décideurs et des chercheurs " (1985: 130). On remarque l'absence de la population. 
Par ailleurs, on doit souligner que la récente réflexion sur la recherche-action est totalement obnubilée par les rapports praticienschercheurs, et quelques exemples illustrent bien cette tendance. Pour Le Gal (1984), toute réflexion sur la recherche-action nous renvoie au rapport binaire théorie-pratique. Face à une telle dichotomie, l'auteur essaie de se situer dans l'entre-deux, en soulignant que
"les chercheurs en sciences sociales ne peuvent nier que leurs travaux se nourrissent, voire s'enrichissent des problèmes issus du terrain de la pratique et les praticiens du secteur social ne peuvent pas plus nier qu'un regard extérieur favorise un éclairage nouveau qui permet souvent de débloquer une situation, de formuler autrement un problème, etc. " (1984: 8). En somme, il estime que le fossé entre ces deux catégories d'acteurs peut être réduit.

De son côté, Autès (1984) estime qu'il faut tenter "à la fois de rapprocher, sans jamais les confondre, les logiques des chercheurs et des praticiens, de nouer un espace de négociation/affrontement ouvert ». De même, pour Martin (1985), l'intérêt de la recherche-action en travail social est de favoriser le rapprochement de différents acteurs avec chacun leur logique spécifique. C'est le cas notamment pour les décideurs politico-administratifs qui visent ainsi à

" mieux saisir et orienter la sphère des services et de la distribution, de mieux coordonner l'économique et le social, de mieux ajuster les politiques sociales ou d'action sociale à leurs cibles. Cette demande du pôle administratif et politique est donc praxéologique, appliquée ou légitimante...» (1985: 134).

Mais c'est aussi le cas des chercheurs en sciences sociales et des professionnels de l'action sociale qui ont manifesté, au cours des dernières années, un intérêt grandissant pour ces nouvelles pratiques de recherche. Ainsi, l'engouement pour la recherche-action dissimule divers enjeux et des intérêts multiples.

En somme, derrière le volontarisme bienveillant et les bonnes intentions se dissimulent d'autres enjeux :

"Ainsi, dans la confusion des rôles de chercheur et de praticien s'exprime implicitement une lutte pour la production du savoir [...] Si le chercheur peut revendiquer de jouer un rôle dans la promotion de l'innovation, ou le praticien dans la production de sens [surtout lorsqu'il s'agit de saisir le sens de sa propre pratique], ceci doit se faire dans le cadre d'une collaboration." (Martin, 1985: 136).

Toutefois, la collaboration dont il est ici question vise plutôt les rapports praticiens/chercheurs. Très bien ; mais où est passée la population? 
Cette question est d'autant plus importante que toute la littérature, soulignent Gélinas et Brière (1985: 128), ne cesse d'insister sur la coopération et le dialogue entre les acteurs. De même, les définitions qui ont circulé au Québec insistent sur la collaboration des divers partenaires. Par exemple, pour la Commission d'étude sur les universités du Québec, la recherche-action c'est :

"essentiellement un travail d'équipe de chercheurs qui alliant la pensée théorique à l'intervention, travaillant avec des groupes extérieurs à leur institution, analysent avec ces derniers les problèmes de leurs milieux, les aident à les percevoir plus nettement et à prendre eux-mêmes en charge les secteurs où se vivent les enjeux majeurs de leur vie collective." (1979).

Pour les participants au colloque sur la recherche-action de Chicoutimi "la recherche-action est un processus dans lequel les chercheurs et les acteurs conjointement, investiguent systématiquement une donnée et posent des questions en vue de solutionner un problème. " (1981). Pour l'équipe éditoriale de la Revue internationale d'action communautaire $(R I A C)$, la recherche-action fait appel à la "capacité de réflexion critique, d'apprentissage qu'un groupe peut manifester sur sa propre action, sa capacité de pouvoir décider par lui-même de façon rationnelle des orientations et des modifications qu'il doit apporter à son projet d'action" (1981). Toutes ces définitions impliquent donc explicitement une volonté réelle d'instaurer un nouveau rapport entre les participants, mais qu'en est-il concrètement?

\section{Quelques pratiques de recherche}

Quant aux travaux de recherche-action en travail social, plusieurs recensions récentes, tant au Québec qu'à l'étranger, indiquent une situation assez similaire à celle que nous venons de décrire pour le champ sanitaire, à savoir une faible participation de la population locale à la démarche. Du ménage à trois (population/praticien/chercheur), on revient au bon vieux couple théorie/pratique, avec le risque d'aboutir à la famille monoparentale si on ne perçoit pas clairement les enjeux. Bref, il y a un fort mouvement qui se dessine actuellement, surtout du côté des universitaires, pour utiliser la recherche-action comme le moyen privilégié pour développer des modèles d'intervention ou comme justification pour se rapprocher de la pratique, tout en allant chercher, à l'occasion, d'importants budgets de recherche. À titre indicatif, sans viser aucunement à l'exhaustivité, nous allons citer quelques exemples, dont nos propres expériences. Un peu comme 
pour le secteur sanitaire, nous parlerons de recherche-action en travail social dans la mesure où il y a présence au sein de l'équipe d'un(e) travailleur(e) social(e). Et comme le champ du travail social se divise en secteurs assez précis, nous allons examiner les recherches effectuées dans deux domaines particuliers : celui de l'intervention en réseau en santé mentale et celui de la gérontologie sociale, en notant pour chacun d'eux les types de recherche qui nous paraissent refléter le courant dominant, et d'autres appartenant à un courant plus minoritaire.

Citons tout d'abord le projet de recherche-action de Bernier (1983) qui visait à mettre au point un modèle d'intervention anti-stress qui soit "standardisé, polyvalent, pertinent pour la pratique des intervenants sociaux " (1983: 216). Il y a également les nombreux travaux autour de l'intervention en réseau dans le champ de la santé mentale (Brodeur et Rousseau, 1984 ; Daher, 1980) de même que ceux de l'équipe du Centre hospitalier Douglas à Montréal (1982). Plus récemment, il y a eu les démarches de recherche-action du Groupe de recherche-action sur les réseaux de soutien et les pratiques institutionnelles (GRARSPI), qui se sont centrées sur les thèmes de la prise en charge par le milieu et le développement de pratiques sociales dites "alternatives" (Mayer, 1985). Un peu dans la même perspective on peut ajouter la rechercheaction féministe qui vise à développer un programme de formation adapté à l'intervention auprès des femmes violentées (Rinfret-Raynor et al., 1985).

Ces récentes recherches ont comme caractéristique principale et commune de s'orienter vers l'élaboration d'un modèle de pratique défini par un collectif chercheur/praticien. Par exemple, si on applique la recherche-action au champ de l'intervention en réseau, Rousseau (1980) souligne qu'il faut toujours se référer au vécu des praticiens avant de théoriser, et qu'il faut aussi organiser le processus de recherche en s'assurant le respect des réseaux primaires; cela signifie plus concrètement " faire accéder à une problématique collective élaborée par le réseau et favoriser chez celui-ci le recours à des solutions autonomes" (1980: 324). Rousseau croit "que le réseau se révèle à l'intervenant dans la mesure où ses actions et ses attitudes favorisent l'émergence du discours du milieu. Il nous faut éviter d'imposer des techniques d'intervention et de recherche irrespectueuses du déroulement spontané et naturel du vécu du réseau » (1980: 324).

Soit, mais comment faire? L'auteur suggère un processus de recherche-action basé sur quatre étapes intimement liées les unes aux autres : le vécu ; le vécu décrit ; le vécu réfléchi ; et, finalement, le vécu conceptualisé. Ceci constitue, selon lui, « un tout cohérent qui permet 
l'émergence d'une connaissance des réseaux primaires liée le plus possible au réel vécu " $(1980: 325)$. Cet effort de conceptualisation est intéressant et louable, nous le reconnaissons, mais nous estimons (pour l'avoir vécu nous-même) qu'en pratique, le "poids » et l'influence des praticiens et des chercheurs dans l'élaboration du modèle d'intervention est sans commune mesure avec celle de la population. Elle demeure essentiellement objet de recherche et elle participe assez peu, pour ne pas dire pas du tout, à l'élaboration de ce modèle de pratique.

Encore ici, quelques exceptions confirment la règle. Mentionnons par exemple la démarche d'un Collectif qui a entrepris, en 1979, une recherche-action sur les besoins en éducation populaire pour le compte d'un regroupement provincial d'associations de familles monoparentales (Lamoureux, 1984). Après avoir relaté les diverses péripéties de la démarche, elle conclut que "malgré les valseshésitations, les demi-échecs, les galvaudages des mots analysant le processus et la signification de la recherche-action militante, malgré le danger toujours présent de développer des théories dites de libération qui confèrent aux intellectuel(le)s la gestion de la pauvreté, la recherche-action militante demeure un excellent moyen de partage du savoir" (1984: 88). Ceci dit, il faut bien reconnaître que la bonne volonté de se mettre au service des groupes populaires ne suffit pas; il faut pouvoir résoudre une foule de problèmes concrets. L'auteure fait deux autres remarques pour insister sur l'importance " de bien articuler la dernière phase de la recherche-action qui est celle de faire en sorte que le milieu se réapproprie réellement l'analyse et les perspectives d'action » (1984: 91). À la fin de son exercice d'évaluation, Lamoureux a l'impression d'être demeurée malgré tout à la surface des choses: «Bien que je ne voulais pas du tout faire l'économie de l'effort de réflexion théorique, je m'aperçois mieux maintenant comment c'est la pratique elle-même qui pose le besoin d'une théorie capable de l'éclairer et de la guider." (1984: 91). Nous sommes totalement en accord avec une telle conclusion car il nous semble que, dans l'état actuel de la recherche-action, la réflexion sur la pratique doit avoir préséance sur la réflexion strictement théorique.

Quant à la recherche traditionnelle en gérontologie sociale, elle est relativement récente et, de ce fait, comporte plusieurs limites. À ce propos, Édith Skutezki-Odier (1985) note que

"la gérontologie sociale a réellement été inventée à la fin des années 50 et dès lors, l'intérêt pour la pauvreté et les problèmes vécus par les personnes âgées dans notre société, ont servi de point de départ à un arsenal important de recherches, dites scientifiques. Cependant, selon certains auteurs, il paraît qu'en recherche traditionnelle ou 
scientifique, il est courant d'étudier les phénomènes reliés au vieillissement sous l'angle des déficits, des diminutions et des pertes fonctionnelles." (1985: 54).

Après un survol des recherches en gérontologie, notamment au Québec, elle conclut que la recherche dans ce secteur "porte malheureusement sur les personnes au lieu d'être à leur service " (1985 : 63).

Pour contrer ces limites, Skutezki-Odier plaide en faveur de la recherche-action afin de susciter la participation des préretraités et des retraités à titre de partenaires égaux à cet effort de recherche car ils " ont tous les prérequis et attributs nécessaires et souhaitables qu'on puisse imaginer qu'un chercheur (ou promoteur) participatif devrait ou pourrait avoir. Ils ont un long vécu à titre d'expérience, avec la sagesse qu'uniquement le parcours des années peut apporter. Ils ont aussi de la vigueur, de la rigueur et du temps» (1985: 64). Dans une perspective similaire, Thiénot (1980) a retracé le processus de recherche-action qui a réuni des groupes locaux de retraités et de préretraités québécois ainsi que des "intellectuels" universitaires. Ce rapprochement a débouché notamment sur la publication de manifestes ainsi que sur la création d'une Association québécoise pour la défense des droits des retraités et des préretraités (A.Q.D.R.). Toujours dans la même perspective, Ivanovic et Sommer (1981) ont raconté une expérience de recherche-action qui offre plusieurs points de similitude avec l'expérience de l'A.Q.D.R. que nous venons d'évoquer. Sans entrer ici dans le détail de l'expérience, soulignons simplement que cette perspective générale a amené le groupe à s'engager dans différentes formes d'action telles que la sensibilisation de l'opinion publique en général et des organisations syndicales et populaires en particulier, la participation à des réunions (clubs de retraités), la rédaction d'articles, l'élaboration d'un projet de réforme des régimes de pension destiné à servir de base de discussion avec les milieux concernés, la mise sur pied et l'expérimentation, dans une entreprise et dans un quartier de la région, d'un programme de préparation à la retraite, etc. En somme, ces pratiques de recherche-action convergentes illustrent l'utilité et la richesse de la participation des gens du milieu au processus de recherche. 


\section{Recherche-action dans le domaine de l'éducation}

Des travaux québécois ont très bien montré les applications et utilisations possibles de la recherche-action dans le domaine de l'éducation. André Morin $(1981,1984)$ a fait le tour des diverses orientations de la recherche-action en éducation, tant au Québec qu'aux États-Unis; Rhéaume (1982) a évoqué le rôle du Ministère de l'éducation du Québec qui sera l'initiateur, au début des années soixante-dix, de plusieurs projets de recherche-action d'envergure. On lancera, par exemple, une vaste enquête-participante (l'OpérationDépart) dans les diverses régions du Québec pour faire l'inventaire des besoins de formation de la population adulte. De même, le projet TÉVEC (1967) et le projet Multi-Media (1971) seront de vastes expériences de télévision éducative pour adultes, à partir d'une pédagogie basée sur les problèmes socio-économiques du milieu et sur une structure d'animation locale devant en favoriser la participation.

Pour Thirion (1981), la recherche-action vise essentiellement à analyser les politiques et les pratiques éducatives: "La rechercheaction vaut d'abord comme analyseur des idéologies déguisées en science et des enjeux socio-politiques liés à la dominance sociale et au contrôle du savoir. " (1981: 647). À vrai dire, cette auteure se situe dans la même perspective que celle développée par Zúñiga (1981) à propos du rôle de la recherche-action. Plus concrètement encore, Thirion analyse l'évolution de pratiques de recherche-action qui visaient à prévenir et à réduire les inégalités devant l'éducation chez les jeunes enfants de milieu populaire : "Ces nouvelles orientations de recherche et d'actions sont ébauchées dans le cadre d'un projet qui encourage des pratiques coopératives et un partage de ressources entre des groupes d'action culturelle et des chercheurs concernés par les inégalités devant l'éducation.» (1981: 652). Dans cette problématique, la recherche-action "transforme l'expérience directe, le vécu des groupes en connaissances suffisamment abstraites pour être reproduites, transposées à d'autres situations, communiquées, confrontées à d'autres démarches » $(1981: 650)$. On aura sans doute compris que cette perspective est essentiellement de nature universitaire, et à part le fait de dire qu'il faut encourager leur participation dans l'école, on remarque qu'il est assez peu question des enfants et des parents dans ce texte.

Mais c'est sans doute l'article de Pourtois (1981) qui illustre le mieux la conception dominante de la recherche-action dans le domaine de l'éducation. Grosso modo, l'auteur s'interroge à savoir « comment les 
pratiques éducatives se conçoivent, s'évaluent et/ou se modifient" (1981: 555). Sur le plan théorique, la recherche-action est définie comme étant principalement de nature praxéologique, "c'est-à-dire science de l'action ou, plus exactement, logique d'action orientée essentiellement vers la connaissance raffinée de la pratique en vue de son optimalisation" (1981: 560). Au plan opérationnel, "élaborer et mener une recherche-action consiste à organiser de multiples séances de Diagnostic Renforçant à propos du changement projeté et/ou réalisé entre les personnes en présence, c'est-à-dire les acteurs [chercheurs et praticiens]" (1981: 561). Suit l'exemple d'une rechercheaction auprès des parents dans les classes maternelles d'un quartier ouvrier belge. En conclusion, il est souligné que

"la recherche-action se conçoit comme un processus d'autodétermination. Le but poursuivi est de susciter l'esprit de recherche (activation du besoin de réussite) et l'implication existentielle (activation des besoins affectifs) en vue de faciliter le changement dans une perspective d'amélioration du sort des acteurs-chercheurs et des membres de la communauté élargie.» (1981: 569.)

Mais concrètement, dans l'article, il est beaucoup plus question des acteurs-chercheurs que des membres de la communauté élargie!

Un peu dans la même perspective, Rondeau (1984) raconte le processus d'institutionnalisation d'une pratique de recherche-action dans un service d'éducation en milieu ouvert, auprès de jeunes délinquants. Toutefois, les acteurs de cette recherche sont essentiellement un groupe de travailleurs sociaux et de chercheurs, et l'auteur analyse leurs rôles respectifs dans les différentes étapes (définition de la tâche, construction des outils, production finale, etc.). Compte tenu de l'insatisfaction des praticiens vis-à-vis leurs pratiques quotidiennes et de leurs conflits internes, ils envisagent la recherche, d'une part comme moyen de "faire changer les choses" et, d'autre part, comme moyen "de médiation entre les membres du groupe". Plus concrètement, l'idée initiale du projet est liée à une double méconnaissance :

"méconnaissance de la "population-cliente", en cela que les praticiens ont une connaissance individualisée de cas, mais ce que l'on "sait" est ignoré par l'autre ; autrement dit, l'équipe en son ensemble n'a pas une vision générale du Savoir. D’autre part, il y a également méconnaissance des pratiques éducatives individuelles, ici l'équipe n'a pas une vision générale du Faire. Cette double méconnaissance semble préjudiciable à un fonctionnement "harmonieux" et "adapté" du service. » $(1984: 60)$.

D'où l'idée d'organiser une recherche "dont l'objectif premier n'est évidemment pas de constituer un travail académique et gratuit, mais 
doit tendre à devenir un support pour la définition précise de notre action et lui apporter un élément supplémentaire de cohésion interne et d'efficacité.» (1984: 58.) Mais au-delà de ces objectifs légitimes, il n'en reste pas moins, qu'encore ici, la "population cliente» demeure objet d'étude et que la recherche semble répondre davantage, en définitive, aux divers besoins des professionnels.

Pour sa part, Morin (1984) résume ainsi le bilan d'une rechercheaction en éducation populaire :

"Lorsque le chercheur est convié à aider un groupe à s'exprimer, à réfléchir et à écrire, il est essentiel que les intervenants sociaux s'impliquent à tous les niveaux du long processus de recherche. II importe de prévoir un contrat ouvert qui permet de se questionner mutuellement sur les significations des actions et des réflexions. De plus [...] le dialogue constant entre le chercheur et l'acteur, tant dans l'action que sur l'action, suppose que cette dernière l'interpelle, remette en question ses théories [...] Par ailleurs, il convient de se rappeler qu'une communication entre auteurs et acteurs qui ne susciterait que de l'agressivité n'apporterait aucun enrichissement au savoir. La recherche-action doit favoriser un modèle d'interaction permettant l'ouverture aux expériences réciproques et à un questionnement constant sur la problématique et les hypothèses de solutions." (1984: 452).

L'auteur dégage de cette expérience un certain nombre de leçons dont les principales sont : 1) la recherche-action exige une négociation qui permet l'implication ; 2) elle nécessite un contrat ouvert ; 3) au-delà d'une simple application de la théorie à la pratique, elle doit viser une quête de significations mutuelles; 4) pour ce faire, elle doit refuser la communication agressive et la contestation aveugle pour s'orienter plutôt vers l'acceptation de l'expérience des autres, etc. Mais l'intérêt de ce compte rendu, c'est de montrer que la volonté d'impliquer la population dans le processus de recherche-action ne va pas sans problèmes pour les divers acteurs impliqués. En effet, si une partie des chercheurs en sciences sociales est assez réfractaire à la rechercheaction, les milieux populaires comme ceux de la pratique se sentent parfois menacés, dans la mesure où la recherche-action vient bousculer les habitudes et les certitudes des uns et des autres.

Quant au courant minoritaire de la recherche-action dans le domaine de l'éducation, il est représenté par l'expérience menée par le groupe "La Maîtresse d'école" (Cadotte et al., 1979), qui a tenté de développer des expériences de pédagogie progressiste à partir de l'université. Ces éducateurs-intervenants ont élaboré un processus de recherche-action des plus intéressants. Le groupe "La Maîtresse d'école" insiste sur le fait que le projet doit aboutir à un résultat 
concret, matérialisable et communicable. Selon lui, c'est d'ailleurs là "la seule condition pour réduire l'éternelle contradiction intellectuel/ manuel et pour éviter que des membres des classes populaires ne s'acculturent, c'est-à-dire ne se coupent de leur groupe d'origine en acquérant certains savoirs qu'ils ne sont pas capables de leur faire partager" (Cadotte, 1979: 100). Cette problématique de la rechercheaction et de la pédagogie progressiste a valu à ce groupe son éviction de l'université à toutes fins utiles. Cette attitude "ferme», c'est le moins que l'on puisse dire, illustre assez bien la "résistance" de certains milieux universitaires à certaines formes de pratique de rechercheaction dans laquelle la population est active.

\section{Conclusion}

Au terme de cette étude, nous retenons les idées suivantes:

$1^{\circ}$ Il semble se dégager un décalage sans cesse croissant entre le discours sur la recherche-action et sa pratique concrète. En effet, si théoriquement la recherche-action veut proposer un nouveau modèle de rapports, c'est-à-dire l'implication de trois acteurs (intervenants, chercheurs et citoyens concernés) dans un processus de recherche ancré dans une réalité sociale ou dans une problématique d'intervention concrète, la réalité est malheureusement toute autre. Comme nous l'avons vu précédemment, la population est encore trop peu présente, en pratique et dans la réalisation des projets de recherche. Malgré un discours qui tente de définir la recherche-action en tant qu'œuvre collective, sa pratique ne semble pas remettre en question le pouvoir entre les différents partenaires. Malgré ses prétentions contraires, elle ne fait pas disparaître comme par enchantement la division sociale du savoir et du pouvoir, car elle s'inscrit et se développe dans une société profondément divisée et inégale (Bolle de Bal, 1981), et la reconnaissance de ce fait permet de mieux situer les enjeux.

$2^{\circ}$ Si la recherche-action, lors de son implantation, fut marquée d'un certain idéalisme voulant que les rôles se métamorphosent, les auteurs contemporains tentent davantage de situer les rôles respectifs dans le respect des différences. Par contre, cette remise en question doit nécessairement passer par la participation active de tous les acteurs impliqués. Si les rôles des chercheurs sont précisés et discutés, en recherche-action, ceux des 
intervenants-praticiens et groupes impliqués le sont beaucoup moins. Les écrits des chercheurs sont relativement muets à cet égard. Par ailleurs, on oublie souvent de considérer la réticence des groupes populaires face aux institutions et leur peur obsessive d'être récupérés. Ce repli sur soi se base sur des expériences malheureuses, mais il ne constitue pas moins un obstacle à l'implantation de la recherche-action. L'expérience québécoise de Morin (1984) le confirme assez bien.

$3^{\circ}$ Pour surmonter ces difficultés, une meilleure reconnaissance des obstacles rencontrés par les groupes s'impose, particulièrement en ce qui concerne l'écriture et la gestion du temps. En effet, la recherche-action implique la production d'un certain savoir, mais Le Boterf (1983) s'interroge : "Qui rend compte de ce savoir? Qui le fait connaître? Qui rédige rapports et articles sur le processus même de recherche-action? Quels sont les codes utilisés (écriture, langage oral, audio-visuel, etc.) dans ces communications?" (1983: 46). Trop souvent, souligne-t-il, la recherche-action demeure dans les mains de ceux qui maîtrisent les codes dominants, surtout celui de l'écriture et, par conséquent, la place des chercheurs professionnels dans ce processus demeure prépondérante.

Pour plusieurs chercheurs (Offredi, 1981 ; Desroches, 1982 ; Morin, 1984), la fonction d'écriture s'avère fondamentale dans le processus de recherche-action. Il s'agit de faire en sorte que les acteurs deviennent des auteurs. Mais l'opération n'est pas aussi simple et c'est plus facile à dire qu'à faire car nous sommes souvent en présence de différences profondes entre les chercheurs et les autres participants au processus de recherche. Ces différences traduisent d'ailleurs des systèmes de valeurs et des modes de pensée différents. Pour leur part, Haramein et Perrenoud (1981) concluent que la question de l'écriture n'est jamais simple dans une recherche-action et que "les textes, surtout s'ils sont écrits essentiellement par les chercheurs, ne sont pas le seul moyen, ni sans doute le meilleur compte rendu d'une recherche-action » (1981: 230).

Par ailleurs, la recherche-action exige du temps, et comme nous le rappelle Le Boterf :

"Les chercheurs professionnels et les experts disposent du temps requis, car il s'agit d'une composante de leur situation professionnelle. Les acteurs, eux, doivent prendre habituellement sur leur temps de non-travail le temps qu'ils consacrent à la recherche-action. L'inégalité dans la possession du capital-temps ne va-t-elle pas induire une inégalité dans le pouvoir de conduire et de contrôler le processus de recherche-action?» (1983: 46). 
Voilà une bonne question, et qui mérite une réponse... concrète si possible ! En attendant, et compte tenu de la dominance des chercheurs (des universitaires pour la plupart), tant au niveau du terrain que de l'écriture, on doit conclure qu'on est encore loin des objectifs de la désinstitutionnalisation du savoir et de désacralisation de la science.

$4^{\circ}$ Plusieurs auteurs soulignent que la spécificité de la rechercheaction réside dans son caractère collectif : "Dans ce type de recherche, la quête de l'objectivation, la prise de distance par rapport à l'objet perçu quotidiennement, la rupture épistémologique ne sont possibles que grâce à la confrontation des points de vue, mieux, des systèmes de représentations des différents acteurs. » (Le Gal et Martin, 1983 : 68). Encore faut-il qu'ils soient tous là ! Il est assez paradoxal que ceux qui visent la promotion d'une science sociale "d'en bas" ou d'une "méthodologie douce" plus près du terrain et des pratiques concrètes aient oublié un des acteurs du processus de la recherche-action : la population. Par contre, nous ne voulons pas tomber non plus dans une utopie volontariste ou populiste et croire que la population peut participer sans problème à toute démarche de recherche-action. Mais ceci est un autre problème qui mériterait plus d'attention.

$5^{\circ}$ Dit en d'autres mots, nous avons trouvé, selon la typologie de Desroches (1982), une nette dominance de la recherche-action d'explication ("recherche-sur") de même que de la rechercheaction d'application ("recherche-pour»), avec toutefois une présence nettement moins grande de la recherche-action d'implication ("recherche-par" ou "recherche-avec" les citoyens ou les acteurs sur le terrain). Cependant, cette typologie peut être remise en cause compte tenu de ce que nous avons constaté, à savoir une nette dominance du modèle de recherche-action avec les professionnels pour l'institution sur la population ("recherche-avec-pour-sur"!). Nous avons bien sûr relevé, dans les divers champs de pratique, des expériences de recherche-action dans lesquelles le lien avec la population est effectivement amorcé ou réalisé avec plus ou moins de succès. Toutefois, il s'agit là d'un modèle nettement minoritaire.

Au bout du compte, nous avons l'impression, et ce sont là des hypothèses pour fins de discussion, que ce modèle minoritaire dépend moins de la bonne ou mauvaise volonté des chercheurs et des praticiens que des conditions extérieures dans lesquelles s'insère cette 
pratique de recherche-action, et notamment le degré de professionnalisation du champ de pratique et le niveau d'organisation des groupes impliqués. Les secteurs où nous avons relevé un certain nombre de recherches-actions réalisées avec la participation de la population locale, par exemple les secteurs de la santé et sécurité au travail dans le champ sanitaire et celui de la gérontologie sociale dans le champ social, sont précisément des secteurs où, d'une part, le taux de professionnalisation semble relativement moins élevé et, d'autre part, où le lien avec des groupes organisés (syndicats, groupes populaires) est plus fort. Seule une analyse plus poussée et plus systématique de la pratique de recherche-action dans les divers secteurs pourra nous permettre d'apporter un début de réponse à ces interrogations.

\section{Notes}

1 Cet article a fait l'objet d'une communication lors du Congrès du Regroupement des unités de formation en travail social du Québec, tenu dans le cadre de l'ACFAS (Montréal, mai 1986).

2 Nous avons seulement considéré les recherches se réclamant formellement de la recherche-action et ayant fait l'objet d'un rapport écrit. Ce recensement s'est fait principalement à partir du Répertoire des recherches en santé du Québec (Gris, 1985) enrichi de quelques publications dont nous avons eu connaissance. Cette façon de procéder, bien que critiquable, nous a fait prendre conscience de la difficulté de recueillir, d'une manière systématique et exhaustive, les expériences de recherche-action.

3 Le poids des professionnels ne se mesure pas, bien entendu, uniquement en caractères quantitatifs. La valeur accordée à la santé et la puissance du modèle médical viennent renforcer ce "poids quantitatif ".

${ }^{4}$ Il est bien évident qu'entre les différents professionnels, il existe un corporatisme, une division des tâches et une hiérarchie constitués autour du pouvoir médical. Mais ceci n'empêche en rien la constitution d'un véritable "front" face aux non-professionnels.

\section{Références bibliographiques}

Autès, M., "Théorie et pratique : une question, un enjeu ", Service social dans le monde, vol. 43, no 3, 1984: 16-21.

BERNIER, D., "L'intervention anti-stress. Une approche pertinente pour la formation des intervenants psychosociaux", Revue canadienne de service social, $1983: 215-227$. 
Bolle De BAL, M., "Nouvelles alliances et reliances: deux enjeux stratégiques de la recherche-action ", Revue de l'Institut de sociologie, no 3, 1981: 573-587.

BOUSQUET, B., "Recherche, quand tu nous tiens... ", Service social dans le monde, vol. 43, no 3, 1984 : 3-7.

BRodeur, C. et R. RousSeAu, L'intervention de réseaux, une pratique nouvelle, Montréal, France-Amérique, 1984.

Cadotte, R., M. Desjardins et al., "La pédagogie progressiste à l'université : l'expérience de la Maîtresse d'école", Revue internationale d'action communautaire, 2/42, 1979 : 95-106.

CARON, D., Le collectif garderies, une expérience d'éducation des adultes en santé communautaire, mémoire de maîtrise, Faculté des sciences de l'éducation, Université de Montréal, août 1982, 143p.

ChAMBAUD, L. et G. RiCHARD, Le projet santé agricole : éléments d'analyse et de réflexion ou les difficultés de l'approche recherche-action dans le réseau de la santé communautaire, St-Jean-sur-Richelieu, Québec, Département santé communautaire de l'Hôpital du Haut-Richelieu, 1984, 49p.

COMmission d'ÉTUde sur les Universités du QuéBec, Rapport du comité de coordination, Québec, Éditeur officiel du Québec, 1979.

Conte, N., M. Loncol et M. Regimbald, "Recherche-action avec le groupe "Alternative-naissance" ", Trans Marge, Cahier I, automne 1985 : 9-33.

CORPET, A., "Collectif d'intervention et mouvements alternatifs", Archives de sciences sociales, de la coopération et du développement, Paris, no 62 , 1982 : 33-72.

DAAB, W. et L. AbenhalM, "Connaissance scientifique et action en santé publique : l'utilité de la recherche dans l'élaboration d'un programme de santé ", Revue canadienne de santé publique, vol. 75, no 5, 1984: 388-392.

DAHER, P., "Les pratiques de réseaux : recherche-action ", Intervention, n० 58 , $1980: 13-24$.

Darveau-Fournier, L., "Bénéficiaires et intervenants, partenaires dans l'amélioration de la qualité de vie : une recherche-action en milieu de soins prolongés ", Service Social, vol. 34, no 1, 1985 : 30-45.

Département de santé communautaire du Centre hospitalier de l'Université Laval (CHUL), Expérimentation d'une intervention visant les pratiques alimentaires de pré-adolescents au déjeuner, 1984, 119p. Resp. : P. Joubert, M.C. Lepage et L. Momassin.

Département de santé communautaire de l'Hôpital Sacré-Cœur, Rechercheaction dans le secteur Saint-René Goupil du quartier Saint-Michel, juin 1982,66p. Resp. L. Colgan, R. Dubreuil, D. Giguère, D. Girouard, F. Ouellet et L. Tremblay.

Département de santé communautaire de l'Hôpital Sainte-Justine, Rapport des visites auprès des familles du Sud-Est Asiatique, été 1981, 66p. Resp. Roger Côté.

Desnoyers, L. et D. Mergler, "Formation et recherche en santé et sécurité au travail ", Revue internationale d'action communautaire, 2/42, 1979 : 11-20.

DESROCHES, H., "Les auteurs et les acteurs. La recherche coopérative comme recherche-action ", Archives des sciences sociales de la coopération et du développement, 1982: 39-64. 
Duвost, J., "Une analyse comparative des pratiques dites de recherche-action", Connexions, no 43, 1984: 8-28.

Duвost, J., "Les critères de la recherche-action ", Pour, no 90, 1983 : 17-21.

DUBOST, J. et O. LUDEMANN, "Un nouveau courant de la recherche-action en Allemagne (R.F.A.) ", Connexions, n² 21, 1977 : 101-114.

Dussault, G., "Les effectifs sanitaires au Québec", Traité d'anthropologie médicale. L'institution de la santé et de la maladie, Québec/Lyon, Presses de l'Université du Québec/Institut québécois de recherche sur la culture/Presses Universitaires de Lyon, 1985 : 587-603.

Ehrenreich, B., "L'objectivité et les femmes", dans: Médecine et société ; les années ' 80 , Montréal, Albert Saint-Martin, 1981 : 81-118.

Équipe d'intervention en réseau du Centre Hospitalier Douglas, Réseau primaire et santé mentale : une expérience de recherche-action, Montréal, Unité de recherche psycho-sociale, Centre de psychiatrie communautaire, Centre hospitalier Douglas, avril 1982.

GélINAS, A. et R. Brière, La recherche-action: ses méthodes, ses outils conceptuels et son cadre d'analyse (Recension des écrits) (tome 1), Rapport de recherche, Conseil québécois de la recherche social, Québec, 1985, $207 p$.

Goyette, G. et M. LesSARD-HéBert, La recherche-action: ses fonctions, ses fondements et son instrumentation, Québec, rapport au Conseil québécois de la recherche sociale, 1985.

Groupe de recherche multidisciplinaire en santé, (G.R.I.S.), Répertoire en santé au Québec. 1980-1984, Université de Montréal, Département d'administration de la santé et Département de médecine sociale et préventive 1985.

Haramein, A. et P. Perrenoud, "Rhapsodie : une recherche-action: du projet à l'acteur collectif», Revue européenne des sciences sociales, n 59, 1981 : 175-231.

Hétu, R., "Quelques pas vers une déprofessionnalisation de la santé au travail ", Revue internationale d'action communautaire, 2/42, 1979: 21-26.

Institut national de la santé et de la recherche médicale, La recherche-action en santé, Paris, La documentation française, 1985.

IVANOVIC, M. et M. SOMmer, "Une collaboration entre des chercheurs et des militants retraités ", Revue internationale d'action communautaire, 5/45, 1981: 131-141.

Lamoureux, J., Quelques significations et enjeux de la recherche-action militante, mémoire de maîtrise, Université de Montréal, 1984.

LE Boterf, G., L'enquête participation en question, Paris, Ligue de l'enseignement et de l'éducation permanente, 1981.

LE BOTERF, G., " La recherche-action : une nouvelle relation entre les experts et les acteurs sociaux ", Pour, no 90, $1983:$ 39-46.

LEFRANÇOIS, R., "Les nouvelles approches qualitatives et le travail sociologique", dans: J.-P. DesLAuRIERs (dir.), La recherche qualitative: résurgence et convergences, Chicoutimi, Université du Québec à Chicoutimi, 1985 : 151-169.

LefRANÇOIS, R., "La recherche sociale comme nécessité ", Revue canadienne de service social, 1985 : 171-185. 
Le Gal, D. et C. Martin, "Une recherche qui se cherche", Pour, no 90, 1983 : 67-83.

LE GAL, D., "Recherche versus travail social", Service social dans le monde, vol. 43, no 3, $1984: 8-15$.

Le Gal, D. et C. Martin, "Crise et conversion dans le champ du savoir ", Revue internationale d'action communautaire, 15/55, 1985 : 9-21.

MARTIN, C., "Recherches ou chercheurs appliqués dans/sur le travail social», Service social dans le monde, vol. 43, no 3, 1984: 40-47.

MARtin, C., "Entre logiques du savoir et de l'action; les voies d'une alternative?", dans: J.-P. DESLAURIERS, (dir.), La recherche qualitative: résurgence et convergences, Chicoutimi, Université du Québec à Chicoutimi, 1985 : 123-141.

MARTIN, C., Les recherches-actions sociales: miroir aux alouettes ou stratégie de qualification, Paris, Ministère des affaires sociales et de l'emploi, 1986, 135p.

MAYER, R., Les pratiques de prise en charge par le milieu: une expérience de recherche-action, École de service social, Université de Montréal, 1985.

Mergler, D. et L. Desnoyers, "Milieu de travail et santé ; éléments d'analyse de la situation québécoise", dans: Médecine et société: Les années '80, Montréal, Albert Saint-Martin, Québec, 1981: 235-248.

MORIN, A., "Quelques orientations de la recherche-action en éducation en Amérique du Nord", Revue de l'Institut de sociologie, no 3, 1981: pp. 529-538.

MORIN, A., "Les modes de communication auteurs-acteurs", dans: L'écriture collective : un modèle de recherche-action, Chicoutimi, Gaëtan Morin Éditeur, 1984 : 445-453.

NAVARRO, V., "Work, ideology, and science : the case of medicine", International Journal of Health Services, vol. 10, no 4, 1980: 523-550.

OFFREDI, C., "La recherche-action : l'intellectuel et son rapport à l'action ", Revue internationale d'action communautaire, 5/45, 1981: 82-89.

POuRtols, J.-P., "Quelques caractères essentiels de la recherche-action en éducation ", Revue de l'Institut de sociologie, n 3, 1981: 555-573.

RHÉAUME, J., "La recherche-action : un nouveau mode de savoir ", Sociologie et sociétés, vol. 14, no 1, 1982: 43-52.

Rinfret-Raynor, M., A. Pâquet-Deehy et G. Larouche, "Violence familiale: évolution actuelle au plan de la recherche, de l'intervention et de la formation ", Revue canadienne de service social, 1985 : 315-331.

RONDEAU, J.-L., "Une action de recherche dans un service d'orientation et d'action éducative en milieu ouvert ", Connexions, n 43, $1984: 55-80$.

Rousseau, R., "Recherche-action et intervention de réseaux», Service social, vol. 29 , no $3,1980: 322-332$.

SKUTEZKI-ODIER, E., "L'émergence d'un nouveau concept gérontologique : la recherche participative", Cahiers de l'ACFAS, n० 27, 1985 : 53-64.

SOULET, M.-H., "La recherche qualitative ou la fin des certitudes", dans: DESLAURIERS, (dir.), La recherche qualitative: résurgence et convergences, Chicoutimi, Université du Québec à Chicoutimi, 1985 : 11-31. 
ThIÉNOT, M., "Du côté des retraités et des travailleurs âgés en lutte», Revue internationale d'action communautaire, 3/43, $1980: 89-100$.

THIRION, A.-M., "La recherche-action: un analyseur des politiques et des pratiques éducatives", Revue de l'Institut de sociologie, $\mathrm{n}^{\circ} 3,1981$ : 645-655.

TROUTOT, P.-Y., "Sociologie d'intervention et recherche-action socio-politique", Revue suisse de sociologie, no 6, 1980: 191-206.

VÉZINA, M., "Travail et prévention", Traité d'anthropologie médicale, L'institution de la santé et de la maladie/Presses de l'Université du Québec/ Institut québécois de recherche sur la culture/Presses universitaires de Lyon, 1985 : 587-603.

WERY, A., "Recherche-action dans les centres de santé mentale", Revue de l'Institut de sociologie, no 3, 1981: 637-645.

Wieviorka, M., "Luttes urbaines: leurs acteurs et les chercheurs", Revue internationale d'action communautaire, 4/44, 1980 : 149-155.

ZOLA, I.K., "Cultes de la santé et méfaits de la médicalisation", dans : Médecine et société : les années '80, Montréal, Albert Saint-Martin, 1981: 31-50.

ZúÑIGA, R., "La recherche-action et le contrôle du savoir ", Revue internationale d'action communautaire, 5/45, 1981: 35-45. 